\title{
Case Report: Can Inhaled Adenosine Attenuate COVID-19?
}

\author{
Bruce D. Spiess ${ }^{1 \star}$, Michael Sitkovsky ${ }^{2}$, Pierpaolo Correale ${ }^{3}$, Nikolaus Gravenstein ${ }^{1}$, \\ Cynthia Garvan ${ }^{1}$, Timothy E. Morey ${ }^{4}$, Brenda G. Fahy ${ }^{1}$, Leslie Hendeles ${ }^{5}$, Thomas J. Pliura ${ }^{6}$, \\ Thomas D. Martin ${ }^{7}$, Velyn $\mathrm{Wu}^{8}$, Corey Astrom ${ }^{4}$ and Danielle S. Nelson ${ }^{8}$ \\ ${ }^{1}$ Department of Anesthesiology, University of Florida College of Medicine, Gainesville, FL, United States, ${ }^{2}$ New England \\ Inflammation and Tissue Protection Institute - Northeastern University, Boston, MA, United States, ${ }^{3}$ Medical Oncology Unit, \\ Covid19 Scientific Task Force, Grand Metropolitan Hospital, Reggio Calabria, Italy, ${ }^{4}$ Department of Anesthesiology, University of \\ Florida School of Medicine, Gainesville, FL, United States, ${ }^{5}$ College of Pharmacy, University of Florida, Gainesville, FL, \\ United States, ${ }^{6}$ Private Practice Emergency Department, Champaign, IL, United States, ${ }^{7}$ Department of Surgery (Cardiac \\ Surgery), University of Florida School of Medicine, Gainesville, FL, United States, ${ }^{8}$ Department of Community Health and Family \\ Medicine, University of Florida, College of Medicine, Gainesville, FL, United States
}

This case report demonstrates a small repetition of the case series carried out in Italy wherein inhaled adenosine was administered to patients experiencing severe and worsening coronavirus disease-2019 (COVID-19). The two cases are important not only because they were the first of their type in the United States, but also because both patients were $\mathrm{DNR} / \mathrm{DNI}$ and were therefore expected to die. Study repetition is vitally important in medicine. New work in pharmacology hypothesizes that adenosine-regulator proteins may play a role in the pathogenesis of COVID-19 infection. Furthermore, adenosine, by interacting with cell receptor sites, has pluripotent effects upon inflammatory cells, is antiinflammatory, and is important in tissue hypoxia signaling. Inhaled adenosine is potentially safe; thousands have received it for asthmatic challenge testing. The effects of adenosine in these two cases were rapid, positive, and fit the pharmacologic hypotheses (as seen in prior work in this journal) and support its role as a therapeutic nucleoside.

Keywords: COVID-19, acute respiratory distress syndrome, ARDS, nebulizer, adenosine, case report

\section{INTRODUCTION}

We discuss two cases that presented with profound respiratory distress a number of days after contracting COVID-19 pneumonia. Both patients had polymerase chain reaction (PCR)-confirmed COVID-19 and both patients had vital signs with tachycardia and respiratory rates $>30$ breaths per minute. Furthermore, each person presented with low peripheral pulse oximetry levels, 85 and 64 , respectively. Patient one was on high-flow oxygen and Patient two was on oxygen when these presenting vital signs were found. Arterial blood gases obtained in Patient one showed a $\mathrm{PaO}_{2}$ of $52 \mathrm{mmHg}$ and in Patient two (after establishment of oxygen therapy), a $\mathrm{PaO}_{2}$ of $48 \mathrm{mmHg}$. These two cases are unique in that both patients were likely to die in a short period of time but both were offered off-label inhaled adenosine as a therapeutic option. Both recovered and these cases are reported herein because of the rapid response seen, as well as the fact that these first-in-theUnited States treatments demonstrate a repetition of groundbreaking work from Italy.

Acute respiratory distress syndrome (ARDS) occurs in 3-5\% of patients with COVID-19 who have undergone varying degrees of supplemental oxygen therapy, sometimes leading to mechanical ventilation (Pascarella et al., 2020; Yang et al., 2020). Prior work published in this journal (Geiger et al., 2020) proposed three hypotheses regarding coronaviruses and adenosine. First proposed was that COVID-19 


\section{Inhaled Adenosine for COVID-19 Hypothesized Mechanisms of Efficacy \& Timing}

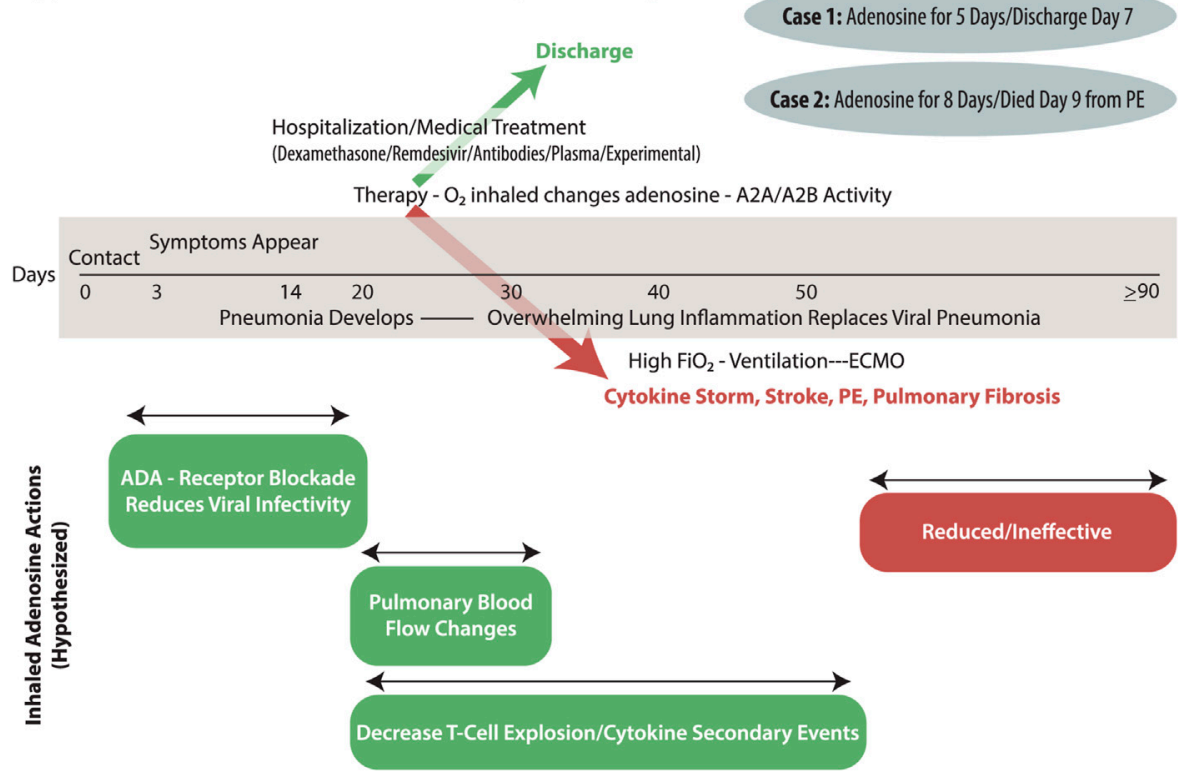

FIGURE 1 | COVID-19 has an individualized and varied timeline from exposure through nasopharyngeal infection, pulmonary spread, pneumonia, and then secondary ARDS. The involvement of oxygen therapy needs to be explored as both a therapeutic and causative player in ARDS as well as the explosive inflammatory cytokine storm of events. The timely use of inhaled adenosine may have efficacy in treatment of COVID-19 infection and secondary complications depending upon the multiple mechanisms by which it could act. Our two patients had a rapid response that almost certainly must have been due to pulmonary blood flow issues, but they recovered from severe COVID-19 ARDS with consecutive days of inhaled therapy. Eventually, and late in the ARDS lung fibrosis continuum, it must be that inhaled adenosine will have reduced effectiveness.

might gain access to pneumocytes through attachment to adenosine kinase $(\mathrm{ADK})$ and adenosine deaminase (ADA) after adhering to viral spike proteins to the angiotensin-converting enzyme (ACE) on cell surfaces. The adenosine-processing transmembrane proteins (ADK and ADA) are coupled with dipeptidyl peptidase 4 (Geiger et al., 2020). Middle Eastern Respiratory Syndrome-CoV (MERS$\mathrm{COV}$ ) has been shown to gain cell entrance through this mechanism of adenosine binding site pore intrusion (Raj et al., 2014; Geiger et al., 2020). COVID-19 and MERS are similar (Raj et al., 2014; Geiger et al., 2020). Extracellular adenosine competes with MERS-CoV for ADK/ADA enzyme binding and transmembrane flow. If such coronaviruses viruses gain intracellular access, they replicate (an adenosine triphosphate/adenosine-consuming reaction) (Geiger et al., 2020). Intracellular replication kills the host cell by degrading energy stores, consuming adenosine, and degrading proteins. Widespread inflammation of pneumocytes leads to an influx of tissue macrophages and killer T-cells, with tremendous secondary inflammation in efforts to ingest and quell the virus, as well as efforts to mop up dead pneumocytes (Geiger et al., 2020). Adenosine is known to down-regulate killer T-cell activity and decrease platelet activation, as well as produce vasodilation and change pulmonary blood flow. Our case report and the Italian series demonstrate clinical efficacy in patients who were in extremis, likely to die, and who were already receiving the best therapy with contemporary regimens of therapy for COVID-19 (Correale et al., 2020). (Figure 1) These cases are compelling and create demand for research, cases series, and randomized controlled trials (timing, dosing, etc.) into efficacy and mechanism (which these cases were unable to investigate).

Both patients (or family) in our case series provided written consent for publication, per University of Florida Institutional Review Board protocol.

\section{CASE 1}

An 89-year-old woman with a history of coronary artery disease, myocardial infarction, polymyalgia rheumatica, temporal arteritis, bronchiectasis, and interstitial lung disease presented with dyspnea and myalgias after 10 days of illness. A computed tomography scan of the lungs revealed extensive reticular infiltrates in the lower lobes intermixed with ground-glass opacities. She was diagnosed with COVID-19 by nasal swab-obtained samples for PCR.

She was admitted and given azithromycin and ceftriaxone for presumed bacterial pneumonia. $\mathrm{Her} \mathrm{SpO}_{2}$ on admission was $94 \%$ on $2 \mathrm{~L}$ nasal cannula (NC) $\mathrm{O}_{2}$ and she was started on remdesivir and dexamethasone (Day 1). On hospital Day 3, increased work of breathing, hypoxia, and worsening non-productive cough occurred, leading to further treatment for superimposed bacterial pneumonia. Her $\mathrm{SpO}_{2}$ was $87 \%$ despite 3.5-6 L/min $\mathrm{O}_{2}$ and a $\mathrm{PaO}_{2}$ of $59 \mathrm{mmHg}$ over Days 3 to 4 . Oxygen support was advanced to a non-rebreathing mask (Day 4; $\mathrm{FiO}_{2} 50 \%$ ) with a resultant $\mathrm{PaO}_{2}$ of 
$78 \mathrm{mmHg}$. The patient and family requested a Do Not Resuscitate/ Intubate status on Day 4. High-flow $\mathrm{NC} \mathrm{O}_{2}\left(60 \mathrm{~L} / \mathrm{min}\right.$ at $\left.70 \% \mathrm{FiO}_{2}\right)$ was accompanied by a respiratory rate $>30$ breaths per minute on Day 7 with an arterial blood gas $\mathrm{PaO}_{2}$ of $59 \mathrm{mmHg}$. She could barely speak. On Day 8, the patient consented to off-label use of inhaled adenosine at $9 \mathrm{mg}$ in $3 \mathrm{~mL}$ of normal saline administered by a vibrating mesh nebulizer (Aerogen, Galway, Ireland) linked to high-flow $21 \% \mathrm{O}_{2}$. Upon administration of adenosine (5 min), her $\mathrm{SpO}_{2}$ improved to $97 \%$ with a concomitant decrease in $\mathrm{FiO}_{2}$, from 75 to $21 \%$. She reported feeling as though her lungs were "opening up," and her respiratory rate went from 30 to $40 / \mathrm{min}$ to 18 to $22 / \mathrm{min}$. She was hemodynamically stable without bronchoconstriction or flushing, and she received another inhaled adenosine treatment $12 \mathrm{~h}$ later and then once daily for the next 4 days without apparent side effects. By Day 2 of adenosine treatment, she was eating and sleeping, and was ambulating by Day 4 . The patient was discharged home 7 days after adenosine commenced. She is now off oxygen and with her family and recovered.

\section{CASE 2}

A 64-year-old man in rural Illinois with chronic myelocytic leukemia and secondary anemia (Hgb 6-7 g/dL) developed COVID-19. His family, members of an Apostolic Christian faith, limit use of traditional medical care, but had a relationship with a neighbor who was a local emergency room doctor. The patient, ill for 1 week, was in extremis when the family called the doctor, who made a home visit and brought a pulse oximeter and rapid COVID test (positive and later confirmed by PCR). When the doctor arrived at the farmhouse, the patient was breathing at 40 breaths per minute and was cyanotic $\left(\mathrm{SpO}_{2} 67 \%\right.$ on room air). He declined hospitalization. The doctor had knowledge of the Italian/UF experiences. He conferred with the UF team, deciding that extreme situations deserve extreme measures. The patient was treated at the farm with oxygen by close-fitting mask, adenosine (9 mg, nebulized), dexamethasone, and antibiotics. Immediately upon treatment, the patient's $\mathrm{SpO}_{2}$ improved to $87 \%$ and his breath rate to 20 per minute. A second dose of adenosine was given at $12 \mathrm{~h}$, and continued for 7 days with twice daily treatments. On Day 4, the patient was eating but was anemic ( $5.8 \mathrm{~g} / \mathrm{dL})$, weak, and was convinced to receive red blood cells and a chest X-ray as an outpatient in an emergency department (bilateral consolidating pneumonia symptomatic of ARDS). On Day 8, he was on 2-5 L $\mathrm{NC}$ oxygen with an $\mathrm{SpO}_{2}$ of $92-95 \%$. On Day 9, the patient walked to his barn to check on farming operations. He felt acutely dyspneic with pleuritic chest pain, was carried to the farmhouse, refused hospitalization, became tachycardic (115-125 beats per minute) with an $\mathrm{SPO}_{2}$ of $70 \%$, and was gasping - an acute change in minutes. $\mathrm{He}$ died quickly with a presumptive diagnosis of pulmonary embolism secondary to COVID-19. The family refused an autopsy.

\section{DISCUSSION}

Our two patients responded to adenosine therapy. We were unable to perform hypothesis testing or adenosine level measurements because these two cases were done as an offlabel clinical effort to save human life and frankly they were not consented for such testing. That these two cases (albeit a very small number) in a different healthcare system provide an apparent parallel effect to that described in Italy (Correale et al., 2020) is important and notable in that repetition of positive results in medicine is vital. Together, our cases, plus the published Italian work (Correale et al., 2020), press the need for hypothesis-based research, toxicologic testing, and prospective randomized trials. This case report cannot satisfy the desire for more mechanistic and pharmacologic information at this time. The immediate improvement we experienced was likely due to pulmonary blood flow changes and not a reduction in inflammation. But the ultimate recovery from ARDs, we believe, has its basis in down-regulation of T-cells, as previously described (Ohta and Sitkovsky, 2001; Sitkovsky et al., 2004; Bours et al., 2006; Aggarwal et al., 2013; NowakMachen et al., 2013; Hatfield et al., 2014; Sharma et al., 2016). The death of the patient in Case 2, presumed a pulmonary embolism, was clearly not an ARDS demise. Yet it must be stated that we cannot rule out an interaction with inhaled adenosine with his COVID-19 disease and/or some unforeseen toxic effect. Clinical trials research needs to be done immediately. Treatment with inhaled adenosine for patients with a $\mathrm{PaO}_{2} / \mathrm{FiO}_{2}$ ratio of $<300$ and at the point of imminent mechanical ventilation could accelerate recovery and save lives. The strengths of such an approach with adenosine are many. The drug is widely available, off patent, and can be easily administered. It appears safe, yet toxicology of repeated daily inhalation has not been rigorously conducted. If the inhaled adenosine is anti-viral early on, then it could open up new and perhaps far-reaching anti-viral technology.

Adenosine, a pluripotent RNA nucleoside, is exported from cells in response to hypoxia-inducible factor-1- $\alpha$-sensed local tissue hypoxia (Ohta and Sitkovsky, 2001; Sitkovsky et al., 2004; Bours et al., 2006; Aggarwal et al., 2013; Nowak-Machen et al., 2013; Hatfield et al., 2014; Sharma et al., 2016; Widysanto et al., 2020). Adenosine, a building block of energy metabolism, is vital to normal physiology, it is anti-inflammatory, and it modulates vascular tone as well as localized tissue blood flow (Ohta and Sitkovsky, 2001; Sitkovsky et al., 2004; Bours et al., 2006; Aggarwal et al., 2013; Nowak-Machen et al., 2013; Hatfield et al., 2014; Sharma et al., 2016; Widysanto et al., 2020). It is vital to energy production and storage through phosphorylated $\mathrm{di}^{-}$and triphosphate molecules in the Krebs cycle. Cells use this energy, but particularly erythrocytes carry a great deal of adenosine and release it at sites of low flow or upon cellular shear and in so doing, autoregulate localized blood flow. Various proteins and nucleoside transporters are important in the constant flux in and out of cells as well as the maintenance, metabolism, and equilibrium of extracellular and intracellular levels of adenosine (Ohta and Sitkovsky, 2001; Sitkovsky et al., 2004; Bours et al., 2006; Aggarwal et al., 2013; Nowak-Machen et al., 2013; Hatfield et al., 2014; Raj et al., 2014; Sharma et al., 2016; Correale et al., 2020; Geiger et al., 2020; Widysanto et al., 2020).

Of interest and fitting with the hypothesis (Geiger et al., 2020) that extracellular adenosine interactions at various receptor sites 
TABLE 1 | Characteristics of two patients treated with inhaled adenosine. Both were elderly/older and both had pre-existing medical conditions.

\begin{tabular}{|c|c|c|}
\hline Variable & Case 1 & Case 2 \\
\hline Age & 89 & 64 \\
\hline Sex & $\mathrm{F}$ & $\mathrm{M}$ \\
\hline Past medical history & $\begin{array}{l}\text { Coronary artery disease, myocardial infarction, polymyalgia rheumatica, } \\
\text { temporal arteritis, primary interstitial pulmonary fibrosis }\end{array}$ & $\begin{array}{l}\text { Chronic myelogenous leukemia treated with } \\
\text { homeopathic medicine }\end{array}$ \\
\hline Allergies & None & None \\
\hline COVID treatment & Remdesivir, dexamethasone, azithromycin & Dexamethasone and azithromycin \\
\hline Oxygen therapy prior to inhaled adenosine & $70 \% \mathrm{O}_{2}$ high-flow nasal cannula at $70 \mathrm{~L}$ & None, room air \\
\hline Respiratory rate prior to adenosine & $25-35$ BPM & 35-45 BPM \\
\hline $\begin{array}{l}\text { Treatment with adenosine ( } 9 \mathrm{mg} \text { in } 3 \mathrm{ml} \\
\text { normal saline at each treatment) }\end{array}$ & BID on Day 1 , Once per day for 4 more days & BID for 7 days \\
\hline
\end{tabular}

are vital in the role of infection and lethality of COVID-19 and MERS-CoV (Geiger et al., 2020) is new work in cystic fibrosis (CF) (Abraham et al., 2021). Adenosine levels have been tested in patients with CF (Abraham et al., 2021) showing that they have abnormally high extracellular adenosine levels due to cellular leakage (10 to 100-fold normal) (Abraham et al., 2021). A patient with CF's propensity for pneumonia and lung supra-infection might make him/her highly susceptible to COVID-19 with an expected very high mortality rate in the population. However, the opposite is true. In multiple countries, no deaths from COVID-19 have occurred in patients with CF. It has been postulated that patients with CF have resistance to COVID-19 because they leak adenosine from inside their cells due to a dysfunction of transmembrane peptidases (where MERS-CoV binds) (Abraham et al., 2021). Furthermore, it is hypothesized that the natural and high concentrations of adenosine in patients with $\mathrm{CF}$ lead to $\mathrm{A} 2 \mathrm{~A}$ and $\mathrm{A} 2 \mathrm{~B}$ receptors recognizing more antiinflammatory signals than those seen in patients without CF. Interestingly, the elderly and those with many underlying chronic disease states have low extracellular adenosine levels and an inability to increase adenosine/ATP export from their cells (Abraham et al., 2021). The CF data, the Italian findings, and our patients fit with the mechanisms and therapeutic options put forward herein and in the prior Frontiers in Pharmacology paper (Geiger et al., 2020).

Local adenosine flux or reduction in the lung prompts vasoconstriction and increases pulmonary shunt. Increased $\mathrm{FiO}_{2}$ administered to treat hypoxia interferes with the natural hypoxia-inducible factor-1- $\alpha$-adenosine axis (Ohta and Sitkovsky, 2001; Sitkovsky et al., 2004; Bours et al., 2006; Aggarwal et al., 2013; Nowak-Machen et al., 2013; Hatfield et al., 2014; Sharma et al., 2016; Dhont et al., 2020; GonzálezDuaarte and Nordcliffe-Kaufmann, 2020; Widysanto et al., 2020). Exogenous administration of adenosine might well allow for restoration of pulmonary adenosine signaling through A2A receptors while relieving systemic hypoxia. "Happy hypoxia" in COVID-19 is noted in some patients who do better with delayed intubation or lower $\mathrm{FiO}_{2}$ (Couzin-Frankel, 2020; Dhont et al., 2020; González-Duaarte and Nordcliffe-Kaufmann, 2020; Widysanto et al., 2020). This phenomenon may be explained by the adenosine axis and $\mathrm{A} 2 \mathrm{~A}$ receptors - that by having permissive relative hypoxia, patients suffering from COVID-19 ARDS have actually increased their endogenous pulmonary adenosine.
Adenosine fits the needs of COVID-19-caused ARDS (Ohta and Sitkovsky, 2001; Sitkovsky et al., 2004; Bours et al., 2006; Aggarwal et al., 2013; Nowak-Machen et al., 2013; Hatfield et al., 2014; Sharma et al., 2016; Widysanto et al., 2020). Extracellular adenosine has powerful cell signaling, anti-inflammatory, anti-platelet, and anti-T cell effects. Normal plasma concentrations range from 30 to $300 \mathrm{nM}$, but with hypoxia, they elevate to $600-1,200 \mathrm{nM}$. Adenosine affects cellular G-protein-linked receptors (A2A and A2B), interfering with toll-like receptor 4-mediated responses that mitigate lymphocyte cytokine responses (Ohta and Sitkovsky, 2001; Sitkovsky et al., 2004; Bours et al., 2006; Aggarwal et al., 2013; Nowak-Machen et al., 2013; Hatfield et al., 2014; Sharma et al., 2016; Widysanto et al., 2020).

Adenosine, a potent platelet inhibitor (Sharma et al., 2016), could potentially affect microthrombosis and diffuse intravascular coagulation. It modulates macrophages, enhancing cellular repair mechanisms and vascular endothelial growth factor (Haskó and Pacher, 2012; Fuentes et al., 2014; Boncler et al., 2019; Widysanto et al., 2020). Reduction of viral loads (as seen in the Italian series) with adenosine has also been reported in West Nile and flaviviruses in conjunction with adenosine usage (Eyer et al., 2017; Tchnesokov et al., 2019). Remdesivir is 1'-cyano-substituted adenosine and shows broadspectrum anti-viral activity (Tchnesokov et al., 2019).

Inhaled adenosine acts differently than intravenous adenosine (Fowler et al., 2000; Fardon et al., 2004; Hendeles et al., 2015a; Hendeles et al., 2015b; Daley-Yates et al., 2020) and does not create a peak blood effect akin to an intravenous bolus. No hemodynamic effects have been described with this technique. Inhaled adenosine has been widely used "off label" as an inhaled diagnostic test of asthma and as a bioassay of inhaled corticosteroid therapy (Fowler et al., 2000; Fardon et al., 2004; Hendeles et al., 2015a; Hendeles et al., 2015b; Daley-Yates et al., 2020). Dosages 10-fold or greater than what we administered have been well tolerated in bronchoprovocation studies (Hendeles et al., 2015a). Inhaled adenosine is so safe that it has been used in thousands of patients-even in pediatric outpatient offices.

Thus far, adenosine appears safe, yet full toxicology of repeated daily inhalation has not been worked out. If inhaled adenosine is anti-viral early on, then it could open up new and perhaps far-reaching anti-viral 
technology. Yet, limitations may exist. At this point, these studies amount to small case series with exciting hypothesized mechanisms. A great deal of effort with randomized controlled trials is required to make sure the observations are validated, and considerable work is yet to be done to confirm the mechanistic hypotheses put forth in this journal and by other work from the NIH. The use of inhaled adenosine is "off label" and not approved by either the European Regulatory Commission or the United States FDA at this time. If this technique is safe and even partially effective before hospitalization, it represents a monumental advance in stopping the spiraling deaths due to COVID-19 ARDS. Clinical practice guidelines could rapidly result from "off label" successes in conjunction with early randomized trials. Toxicology efforts for repetitive inhaled adenosine are under appeal to funding agencies. An Investigational Drug Application has been applied for at the FDA, with toxicology being needed. Future work for other ARDS events beyond COVID-19 are compelled to be performed with this technology if continued encouraging results accrue. The multiple mechanistic hypotheses (Geiger et al., 2020) of inhaled adenosine efficacy compel rigorous exploration.

Table 1 provides a snapshot of treatment for both patients.

\section{Patient Perspective}

Both patients and their families expected death within hours or a day. They were approached by physicians and the therapy was discussed frankly with the perspective that this had been tried in Italy with some success. One family was quite sophisticated in their medical background and the other less so but a basic summary of the science behind why an adenosine inhalant might work was presented. Neither patient could concentrate or comprehend the scientific discussion but both were given as much information as they could attend and take in. Respiratory distress and impending death for both patients may well have played a psychological part in their immediate and willing consent to undergoing the inhalant treatment. Neither patient was able to form sentences, speak, or converse with the physicians prior to the first treatment. Within $5 \mathrm{~min}$ of inhaled adenosine, Patient one expressed (later): "I could feel my lungs opening up and I could breathe again." Indeed, by the end of the first adenosine treatment, she was able to speak in short but full sentences. Similarly, Patient two noted that he could speak and converse with his physician after the first treatment. He could now sit up in a chair and "catch" his breath. Each patient steadily improved each day of treatment. By Day 4, Patient one was eating and by Day 5 , Patient two

\section{REFERENCES}

Abraham, E. H., Guidotti, G., Rapaport, E., Bower, D., Brown, J., Griffin, R. J., et al. (2021). Cystic Fibrosis Improves COVID-19 Survival and Provides Clues for Treatment of SARS-CoV-2. Pruigneic Signaling, 1-12. In press. doi:10.1007/ s11302-021-09771-0 was ambulating, eating, and interacting with his family. Notably, Patient two underwent treatment at his home, interacting with his family the entire time of treatment. Patient one went home on Day 7 and has been communicative with her team, expressing thanks for being alive and for the adenosine therapy. Patient two died on Day 9 after therapy, but he died doing something he wished: farming and interacting with his sons in his barn. His family expressed deep gratitude to the treating physician for not only trying something off label, but they also felt their father's dramatic recovery was a blessed event. Both patients and families noted that the adenosine inhalation was easy, non-painful, and created both immediate and slowly improving conditions.

\section{DATA AVAILABILITY STATEMENT}

The original contributions presented in the study are included in the article/Supplementary Material, further inquiries can be directed to the corresponding author.

\section{ETHICS STATEMENT}

Ethical review and approval was not required for the study on human participants in accordance with the local legislation and institutional requirements. The patients/participants provided their written informed consent to participate in this study. Written informed consent was obtained from the individual(s) for the publication of any potentially identifiable images or data included in this article.

\section{AUTHOR CONTRIBUTIONS}

BS, NG, CG, LH, TP, VW, and DN all variously contributed to the care of the patient, research of background material and relevant literature, drafting the article, and revision of the article. All authors approved the final version to be published.

\section{ACKNOWLEDGMENTS}

The authors wish to thank the following people for their support in treatment of these patients and for preparation of this article. Unfortunately, the type of article does not allow for authorship for all but contributions from each were many: $\mathrm{MS}, \mathrm{PhD}, \mathrm{PC}$, $\mathrm{MD}, \mathrm{PhD}, \mathrm{TM}, \mathrm{MD}, \mathrm{BF}, \mathrm{MD}, \mathrm{TM}, \mathrm{MD}$, and CA, ELS.

Aggarwal, N. R., D’Alessio, F. R., Eto, Y., Chau, E., Avalos, C., Waickman, A. T., et al. (2013). Macrophage A2A Adenosinergic Receptor Modulates OxygenInduced Augmentation of Murine Lung Injury. Am. J. Respir. Cel Mol Biol 48, 635-646. doi:10.1165/rcmb.2012-0351OC

Boncler, M., Wzorek, J., Wolska, N., Polak, D., Watala, C., and Rozalski, M. (2019). Adenosine Receptor Agonists Deepen the Inhibition of Platelet Aggregation by P2Y12 Antagonists. Vasc. Pharmacol. 113, 47-56. doi:10.1016/j.vph.2018.11.005 
Bours, M. J. L., Swennen, E. L. R., Di Virgilio, F., Cronstein, B. N., and Dagnelie, P. C. (2006). Adenosine $5^{\prime}$-triphosphate and Adenosine as Endogenous Signaling Molecules in Immunity and Inflammation. Pharmacol. Ther. 112, 358-404. doi:10.1016/j.pharmthera.2005.04.013

Correale, P., Caracciolo, M., Bilotta, F., Conte, M., Cuzzola, M., Falcone, C., et al. (2020). Therapeutic Effects of Adenosine in High Flow 21\% Oxygen Aereosol in Patients with Covid19-Pneumonia. PLoS One 15, e0239692. doi:10.1371/ journal.pone.0239692

Couzin-Frankel, J. (2020). The Mystery of the Pandemic's 'happy Hypoxia'. Science 368, 455-456. doi:10.1126/science.368.6490.455

Daley-Yates, P., Brealey, H., Thomas, S., Austin, D., Shabbir, S., Harrison, T., et al. (2020). Therapeutic index of Inhaled Corticosteroids in Asthma: a DoseResponse Comparison on Airway Hyperresponsiveness and Adrenal axis Suppression. Br. J. Clin. Pharmacol. 87 (2), 483-493. doi:10.1111/ dcp.14406(Epub ahead of print)

Dhont, S., Derom, E., Van Braeckel, E., Depuydt, P., and Lambrecht, B. N. (2020). The Pathophysiology of 'happy' Hypoxemia in COVID-19. Respir. Res. 21, 198. doi:10.1186/s12931-020-01462-5

Eyer, L., Zouharová, D., Širmarová, J., Fojtíková, M., Štefánik, M., Haviernik, J., et al. (2017). Antiviral Activity of the Adenosine Analogue BCX4430 against West Nile Virus and Tick-Borne Flaviviruses. Antiviral Res. 142, 63-67. doi:10.1016/j.antiviral.2017.03.012

Fardon, T. C., Fardon, E. J., Hodge, M. R., and Lipworth, B. J. (2004). Comparative Cutoff Points for Adenosine Monophosphate and Methacholine challenge Testing. Ann. Allergy Asthma Immunol. 93, 365-372. doi:10.1016/S1081-1206(10)61396-8

Fowler, S. J., Dempsey, O. J., Sims, E. J., and Lipworth, B. J. (2000). Screening for Bronchial Hyperresponsiveness Using Methacholine and Adenosine Monophosphate. Am. J. Respir. Crit. Care Med. 162, 1318-1322. doi:10.1164/ajrccm.162.4.9912103

Fuentes, E., Pereira, J., Mezzano, D., Alarcón, M., Caballero, J., and Palomo, I. (2014). Inhibition of Platelet Activation and Thrombus Formation by Adenosine and Inosine: Studies on Their Relative Contribution and Molecular Modeling. PLoS One 9, e112741. doi:10.1371/journal.pone.0112741

Geiger, J. D., Khan, N., Murugan, M., and Boison, D. (2020). Possible Role of Adenosine in COVID-19 Pathogenesis and Therapeutic Opportunities. Front. Pharmacol. 11, 594487. doi:10.3389/fphar.2020.594487

González-Duaarte, A., and Nordcliffe-Kaufmann, L. (2020). Is "Happy Hypoxia" in COVID-19 a Disorder of Autonomic Interoception? A Hypothesis. Clin. Auton. Res. 30, 331-333. doi:10.1007/s10286-020-00715-Z

Haskó, G., and Pacher, P. (2012). Regulation of Macrophage Function by Adenosine. Arterioscler Thromb. Vasc. Biol. 32, 865-869. doi:10.1161/ ATVBAHA.111.226852

Hatfield, S. M., Kjaergaard, J., Lukashev, D., Belikoff, B., Schreiber, T. H., Sethumadhavan, S., et al. (2014). Systemic Oxygenation Weakens the Hypoxia and Hypoxia Inducible Factor 1a-dependent and Extracellular Adenosine-Mediated Tumor protection. J. Mol. Med. 92, 1283-1292. doi:10.1007/s00109-014-1189-3

Hendeles, L., Daley-Yates, P. T., Hermann, R., De Backer, J., Dissanayake, S., and Horhota, S. T. (2015). Pharmacodynamic Studies to Demonstrate Bioequivalence of Oral Inhalation Products. AAPS J. 17, 758-768. doi:10.1208/s12248-015-9735-7

Hendeles, L., Khan, Y. R., Shuster, J. J., Chesrown, S. E., and Abu-Hasan, M. (2015). Omalizumab Therapy for Asthma Patients with Poor Adherence to Inhaled Corticosteroid Therapy. Ann. Allergy Asthma Immunol. 114, 58-62. e2. doi:10.1016/j.anai.2014.10.012
Nowak-Machen, M., Schmelzle, M., Hanidziar, D., Junger, W., Exley, M., Otterbein, L., et al. (2013). Pulmonary Natural Killer T Cells Play an Essential Role in Mediating Hyperoxic Acute Lung Injury. Am. J. Respir. Cel Mol Biol 48, 601-609. doi:10.1165/rcmb.2012-0180OC

Ohta, A., and Sitkovsky, M. (2001). Role of G-Protein-Coupled Adenosine Receptors in Downregulation of Inflammation and protection from Tissue Damage. Nature 414, 916-920. doi:10.1038/414916a

Pascarella, G., Strumia, A., Piliego, C., Bruno, F., Del Buono, R., Costa, F., et al. (2020). COVID-19 Diagnosis and Management: a Comprehensive Review. J. Intern. Med. 288, 192-206. doi:10.1111/joim.13091

Raj, V. S., Smits, S. L., Provacia, L. B., van den Brand, J. M. A., Wiersma, L., Ouwendijk, W. J. D., et al. (2014). Adenosine Deaminase Acts as a Natural Antagonist for Dipeptidyl Peptidase 4-mediated Entry of the Middle East Respiratory Syndrome Coronavirus. J. Virol. 88, 1834-1838. doi:10.1128/ JVI02935-13

Sharma, A. K., LaPar, D. J., Stone, M. L., Zhao, Y., Mehta, C. K., Kron, I. L., et al. (2016). NOX2 Activation of Natural Killer T Cells Is Blocked by the Adenosine A2AReceptor to Inhibit Lung Ischemia-Reperfusion Injury. Am. J. Respir. Crit. Care Med. 193, 988-999. doi:10.1164/rccm.201506-1253OC

Sitkovsky, M. V., Lukashev, D., Apasov, S., Kojima, H., Koshiba, M., Caldwell, C., et al. (2004). Physiological Control ofImmune Response and Inflammatory Tissue Damage byHypoxia-Inducible Factors and Adenosine A2A Receptors. Annu. Rev. Immunol. 22, 657-682. 10.1146/annurev. immunol.22.012703.104731

Tchnesokov, E. P., Feng, J. Y., Porter, D. P., and Götte, M. (2019). Mechanism of Inhibition of Ebola Virus RNA-dependent RNA Polymerase by Remdesivir. Viruses 11, 326. doi:10.3390/v11040326

Widysanto, A., Wahyuni, T. D., Simanjuntak, L. H., Sunarso, S., Siahaan, S. S., Haryanto, H., et al. (2020). Happy Hypoxia in Critical COVID-19 Patient: A Case Report in Tangerang, Indonesia. Physiol. Rep. 8, e14619. doi:10.14814/ phy2.14619

Yang, X., Yu, Y., Xu, J., Shu, H., Xia, J. a., Liu, H., et al. (2020). Clinical Course and Outcomes of Critically Ill Patients with SARS-CoV-2 Pneumonia in Wuhan, China: a Single-Centered, Retrospective, Observational Study. Lancet Respir. Med. 8, 475-481. doi:10.1016/S2213-2600(20)30079-5

Conflict of Interest: The authors declare that the research was conducted in the absence of any commercial or financial relationships that could be construed as a potential conflict of interest.

Publisher's Note: All claims expressed in this article are solely those of the authors and do not necessarily represent those of their affiliated organizations, or those of the publisher, the editors and the reviewers. Any product that may be evaluated in this article, or claim that may be made by its manufacturer, is not guaranteed or endorsed by the publisher.

Copyright (C) 2021 Spiess, Sitkovsky, Correale, Gravenstein, Garvan, Morey, Fahy, Hendeles, Pliura, Martin, Wu, Astrom and Nelson. This is an open-access article distributed under the terms of the Creative Commons Attribution License (CC BY). The use, distribution or reproduction in other forums is permitted, provided the original author(s) and the copyright owner(s) are credited and that the original publication in this journal is cited, in accordance with accepted academic practice. No use, distribution or reproduction is permitted which does not comply with these terms. 